that small-pox selected the most insanitary districts for its field of operation in Glasgow.

\section{Hospital Statistics.}

Referring now to experience of small-pox and vaccination in Belvidere Hospital, Dr. Chalmers's Table VI teaches the

TABLE VI.-Glasgow.-Small-pox, 1900-1.-Return as to Vaccination of All Cases Treated in Belvidere Small-pox Hosptal from April 1st, 1900, to June 30th, 1901.

\begin{tabular}{|c|c|c|c|c|c|c|c|c|c|c|c|c|}
\hline \multirow{2}{*}{ Age. } & \multicolumn{3}{|c|}{ Vaccinated. } & \multicolumn{3}{|c|}{$\begin{array}{l}\text { Unvaccin- } \\
\text { ated. }\end{array}$} & \multicolumn{3}{|c|}{$\begin{array}{c}\text { Vaccination } \\
\text { Doubtful. }\end{array}$} & \multicolumn{3}{|c|}{ Total. } \\
\hline & 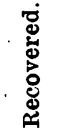 & ه্] & 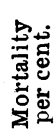 & 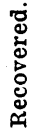 & 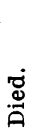 & 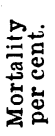 & 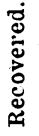 & ه্త & 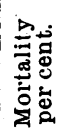 & 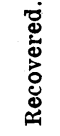 & 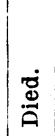 & 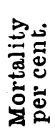 \\
\hline Years. & & & & & & & & & & & & \\
\hline $\begin{array}{cc}0-5 \cdots & \cdots \\
5-10 & \cdots\end{array}$ & $\begin{array}{r}2 \\
3^{1}\end{array}$ & $\underline{r}$ & $33 \cdot 3$ & $\begin{array}{l}18 \\
\text { ro }\end{array}$ & $\begin{array}{r}36 \\
2\end{array}$ & $\begin{array}{l}66.6 \\
16.7\end{array}$ & $\begin{array}{l}3 \\
2\end{array}$ & 二 & $\overline{-}$ & $\begin{array}{l}23 \\
43\end{array}$ & $\begin{array}{r}37 \\
2\end{array}$ & $\begin{array}{r}61.7 \\
4.4\end{array}$ \\
\hline $10-15 \ldots$ & $9^{2}$ & I & I.I & ro & 4 & 28.6 & $\mathbf{I}$ & $\mathbf{I}$ & 50.0 & 103 & 6 & $5 \cdot 5$ \\
\hline $5-20 \ldots$ & $13 \mathrm{I}$ & - & - & 4 & 2 & $33 \cdot 3$ & $\mathbf{I}$ & I & 50.0 & $13^{6}$ & 3 & 2.2 \\
\hline $20-25 \ldots$ & 242 & 12 & 4.7 & 2 & 4 & 66.6 & $\mathbf{I}$ & 2 & 66.6 & 245 & 18 & 68 \\
\hline $25-35 \ldots$ & 584 & 42 & 6.7 & 6 & 5 & 45.8 & 4 & 9 & 69.2 & 594 & $5^{6}$ & 8.6 \\
\hline $35-45 \ldots$ & $29 \mathrm{I}$ & $5 \mathrm{~T}$ & 14.9 & 8 & 4 & $33 \cdot 3$ & 5 & 6 & 54.5 & 304 & $6 \mathbf{r}$ & 16.7 \\
\hline $45-55 \ldots$ & $9 \mathrm{r}$ & 26 & 22.2 & $\mathbf{I}$ & 6 & 85.7 & 3 & 4 & $57 . \mathrm{I}$ & 95 & $3^{6}$ & 27.5 \\
\hline $55-65 . .$. & 18 & 13 & $4 \mathrm{r} .9$ & - & - & - & I & $\mathbf{r}$ & 50.0 & 19 & 14 & 42.4 \\
\hline 65 and over & 10 & 4 & 28.6 & - & 一 & 一 & - & I & 100.0 & ro & 5 & $33 \cdot 3$ \\
\hline All ages ... & 1,492 & ;0 & 9.1 & 59 & 63 & 5 ז. 6 & $2 \mathrm{I}$ & 25 & $54 \cdot 3$ & $\mathbf{I}, 572$ & 238 & 13.1 \\
\hline
\end{tabular}

This table includes cases from beyond city boundaries.

usual lessons (I) that small-pox among the vaccinated is now adays mainly a disease of adults, because children are protected by primary vaccination, and renewal of protection is greatly neglected; (2) that among the unvaccinated small-pox is still in great measure a disease of the young as it was in pre-vaccination times; and (3) that the fatality-rate among the vaccinated is at all ages much less than among the unvaccinated, and that this difference is more striking in children because of the recency of their vaccination. It will be observed from the table that two deaths occurred among 258 vaccinated persons under 20 years of age. One of these two was between ro and 15 years old. The other was a child of 2 years old, "whose vaccination mark was 0.04 of a square inch in area and was glazed and not foveated."

The Scottish Vaccination Act of 1863 came into force in 1864 . In Glasgow there was an epidemic in 1855-57. At that time 88.8 of the small-pox deaths occurred in children under to years old, and only II.2 per cent. in persons over that age. Then Glasgow in 1870.72 had its share of the pandemic of the early Seventies. By that time the Vaccination Act had had from 6 to 8 years in which to protect children born since 1863 . and the percentage of the total small-pox deaths contributed by children under 1o years of age was reduced from the 88.8 per cent. of $1855-57$ to 38.5 per cent. in $1870 \cdot 72$. For many years the amount of default of primary vaccination in Glasgow has averaged only about 3 per cent., so that the recent epidemic found the great bulk of the youthful population primarily vaccinated. That being so, the children under ro years of age, who contributed 88.8 per cent. of the total small-pox deaths in the first of these three epidemics, and 38.5 per cent. in the second epidemic, have now (in 1900-1) contributed only 16.4 per cent., no less than 83.4 per cent. of the cases being over io years old. There can be no doubt whatever that if the average efficiency of primary vaccination in Glasgow, as represented by number and area of marks, had been as high as it ought to be, the contribution made by children under ro would have been a good deal less than even 16.4 per cent.

Hospital Influence.

An important question which we can do no more than mention here is that of hospital influence in the spread of small-pox in Glasgow. In the epidemic of $1870-2$ Glasgow treated its small-pox in Parliamentary Road Hospital, and at that time there was a great aggregation of cases of the disease in the population surrounding that institution. Belvidere Hospital, where the disease has been treated in recent years, is in the east end of the city, and the eastern division has had very much more than its share of small-pox cases: Thère can be no doubt that this adds another to the nany wellknown examples of the influence of small-pox hospitals in spreading the disease in the surrounding population. The Corporation of Glasgow will, it may be assumed, give very serious consideration to the evidence adduced by Dr. Chalmers on the evil effects of Belvidere Hospital during the late epidemic. What remedy they may ultimately adopt, we know not. Meantime, however; we would desire to point out this to them. Among the 400,000 persons recently revaccinated there was not a single case of small-pox. If the remaining portion of the city had been similarly protected there would have been no case of small-pox in Belvidere, and not a single hospital bed would have been required for the treatment of that disease. This does not mean that all the 270,000 of population over 5 years old not recently revaccinated should have been revaccinated at the time of the epidemic. Of the total census population 79,821 were between 5 and ro years old. If primary vaccination were done as efficiently in Glasgow as it ought to be done, these would be excluded from any need for revaccination, and this would make a reduetion of the 270,000 population in question to about 190,000. Very many of these had already been revaccinated at some time or other, and we have seen that they contributed only 19 cases to the epidemic. In short, if all had been revaccinated only once in their lives there would have been no epidemic. $A s$ it is, those who have taken the trouble to protect themselves have also had to contribute their full share to the Belvidere Hospital expenses, and if new hospitals are to be built they will be similarly mulcted.

Very soon the subject of vaccinal legislation in England must again come before Parliament. If Glasgow wants to get rid finally of all the worry and of very neariy all the expense of small-pox hospitals, it should urge its representatives in Parliament to support the policy of getting revaccination at adolescence put on the same legislative footing as primary vaccination of infants, so that exactly the same pressure may be brought to bear on revaccination of children about to leave school as on the vaccination of infants within six months of their birth.

\section{VACCINATION WITH GLYCERINATED CALF LYMPH.}

By ALBERT E. COPE, M.D.Durh., M.B.Lond., D.P.H.

While it máy be given to few to explore the pathology and bacteriology of small-pox and vaccination, or to investigate their history and statistical relations, there are two questions of the utmost practical importance, and of very general interest, which recent legislation has brought into prominent relief. What method should we adopt, and what results may we observe, in the employment of glycerinated calf lymph for human vaccination and revaccination?

The Vaccination Order of 1898 has wisely contented itself with laying down certain general principles, leaving it to the initiative of each vaccinator to apply them. Thus the public vaccinator is instructed that "Vaccination should at every stage be carried out with aseptic precautions. These should include (I) the cleansing of the surface of the skin before vaccination; (2) the use of sterilized instruments; and (3) the protection of the vaccinated surface against extraneous infection both at the performance of the operation and on inspection of the results."

It will be my object to briefly outline some of the methods which may be adopted, and some of the results which may be observed, in the hope that others may be stimulated to give the benefit of their experience, and that an increasing simplicity in our methods may yield more uniformity in our results.

We will suppose then that a child is before us for vaceination. Our first duty is to assure ourselves that the child is fit to be vaccinated. Generally speaking the mother will be quick to let us know of any abnormal condition; but it is none the less important to confirm our general impression of the well-being of the child by an examination of the skin, especially behind the ears and in the napkin area; for evidenees 
of intertrigo or flexural eczema; excluding also condylomata or fissures round the anus. Extensive lichen urticatus or strophulus, especially when associated, as is so frequently the case, with digestive disorder will lead us to postpone vaccination, as also will any impetigo or syphilitic roseola. Elevation of temperature will contraindicate vaccination, whether due to bronchial or intestinal catarrh or other cause, and infantile atrophy or marasmus, it being a safe rule not to vaccinate a child which is losing weight. It will also be necessary to postpone the operation if there is any infectious disease in the family, and wise to do so even if there should be danger of contamination from pus of mammary abscess or ulceration of the leg.

Having examined the child and its surroundings, we have next to cleanse the arm. The morning bath has already come to our help in this respect, but is not sufficient, and we proceed ourselves to wash over the outer aspect of the left arni, which should have been taken completely out of the clothing. What can we use ? Either water, or lotion, or spirit. If water, then we get it from the kettle which has just boiled, or we boil a little ourselves in one of the compact little appliances which all instrument makers keen, or we carry a bottle of freshly boiled water in our bag. We use with the water some form of soap, either plain, or, better, antiseptic, and either in cake or in liquid form. Thus the terebene liquid soap, the ether or ether-mercurial soap, the mercurial so-called germicide soaps, and, best of all, the 20 per cent. carbolic acid soap, as being detergent, antiseptic, and greaseremoving, have all their advocates. The washing should be done with aseptic or antiseptic wool or tow, and any excess of antiseptic should be removed by washing off with plain boiled water, the area being then dried with another pledget of wool. Instead of soap and water we may use weak antiseptic lotions, mercurial carbolic formalin, listerine, but we have to be careful in the use of mercurials, as experience with calves and in human vaccination shows that it is difficult to remove any excess from the skin, and there is danger of the vaccine being inhibited.

In the case of infants it usually will suffice to wash vigorously with rectified spirit, and then dry with pledgets of boric wool or wood wool. This is detergent, and removes grease, is mildly antiseptic, and determines a flow of blood to the surface of the skin. which is desirable for the rapid absorption of the virus. Methylated spirit is objectionable because of smell, and as only small quantities are required the difference in cost is unimportant. In the case of revaccinations, I prefer myself a double washing-first with the carbolic soap and water, then with spirit, and lastly drying, all with boric acid wool pledgets. The spirit is conveniently carried in a bottle with an indiarubber cork, perforated for the reception of a fitting such as is used in ordinary scent bottles.

The skin being prepared, we must choose an instrument. Here, also, there has appeared to be scope for considerable ingenuity, but ingenuity counts for nothing beside safety, simplicity, and efficiency. The various forms of tortoiseshellhandled bleeding-lancet vaccinators are still largely used, though metal handles which can be detached and sterilized completely by boiling may conveniently be substituted.

What are the requisites for scientific vaccination?-

1. An instrument which can be completely sterilized. This puts out of court all the elaborate automatic vaccinators, and many of those with projecting needles. The sterilizing may be by flame, which is in all cases preferable. The temper of steel will be injured, but as we do not need a cutting instrument so much as a scratching one this will be of little consequence. With our spirit bottle, a pledget of wool, and a match stalk, or even the empty capillary tube to make a torch, the process is easy. If boiling is preferred, soda or borax may be added to the water so as to prevent rusting. Antiseptic lotions may be employed instead. The very best vaccinating instrument from this point of view is the palatinum iridium lancet or needle, which may be flamed a thousand times and yet be as bright and almost as good as at the beginning.

2. An instrument with one point. Nothing is so free from precision as vaccination with an instrument, however easily sterilized, which has three or four points; and nothing is so likely to offend the maternal instinct as the sight of four groups of two or three vesicles which she speaks of as " vacci- nation in ten places," a result which it is difficult to avoid in vaccination with such an appliance. The success which it has achieved in the hands of many is simply an evidence of skill on the part of the vaccinator, just as one man may succeed in driving a four-in-hand where another would lamentably fail with a pony and trap.

3. An instrument with a good handle. The vaccinator who has much to do will avoid the needle or pin vaccinator, although it is easily sterilized or thrown away. He does not want to add to the already formidable list of diseases, a "vaccinator's" cramp.

My vote then is given for a platinum double-edged lancet similar in shape to a Paget's abscess knife, with a steel handle, such as is used for ophthalmic knives, all in one piece, the blade sterilized by spirit flame. One special advantage of this type of instrument is that the blade may be made white hot in half a minute, and the parent may see the sterilizing before her eyes. Now, as for the emptying of the capillary tube. For the ordinary tube containing sufficient for one vaccination, and of about 1 to $1.5 \mathrm{~mm}$. external diameter, nothing is better than the pear shaped solid red rubber boilable ejector with funnel-shaped perforation. The tube is

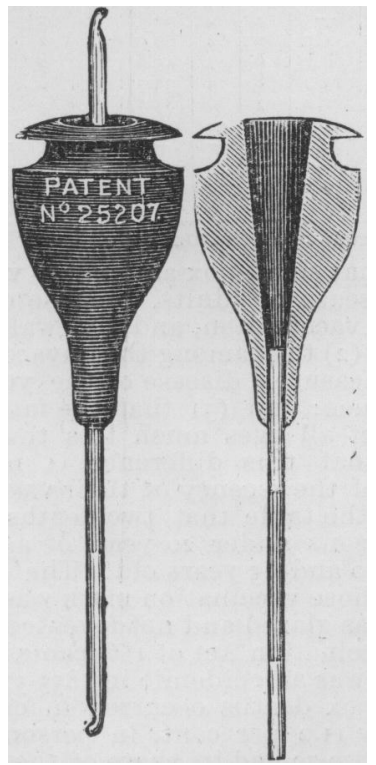

Vaccine ejector (Montague, New Bond Street).

passed through the perforation, both ends broken off between finger and thumb of each hand (or by special breaker), any fragments of broken glass released from the ends; the tube is then drawn on till it becomes the stalk of the pear, the pear is held between first and second finger of left hand, thumb over wide end, when pressure of the thumb compresses air in funnel, and drives out lymph on to the lancet. The same ejector may be used for much wider tubes, but, is not so satisfactory if the external diameter is much less than $1 \mathrm{~mm}$. Several similar ejectors are made in which a small rubber ball takes the place of the funnel-perforated solid rubber, others on the principle of a bellows with verv fine rubber tubing on the nozzle, or a hypodermic syringe or fountain pen filler with similar fitting.

It is better not to blow the lymph on to the skin but on to the lancet, and then place the lymph on the cleansed arm in four places in the form of a square or diamond, with the deltoid insertion in the centre, the sides of the square being about 2 to 2.5 c.cm. All is now ready for the actual vaccination. The operator's left hand ispassed under, and holds the arm firmly, putting the skin on the outer aspect on the stretch. Holding the lancet recommended, as a pen, but somewhat more perpendicularly, the blade being used in the cutting direction and not so as to turn the point, two or three light scratches $5 \mathrm{~mm}$. long are made through each droplet of lymph, and with the flat of the blade the lymph is gently pressed into the scratches. It is extremely rare for blood to flow, although 


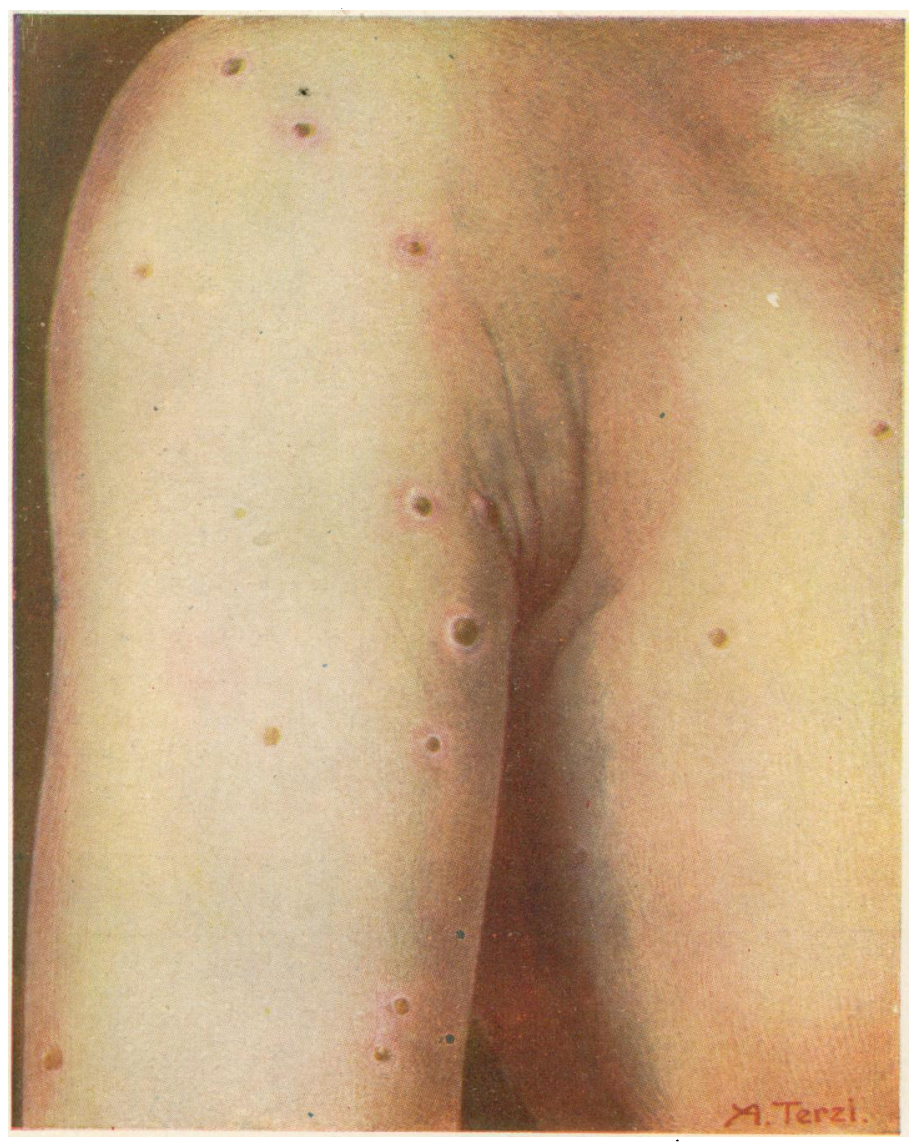

Fig. 1. - SMALL-POX MODIfIED Seventh day of eruption.

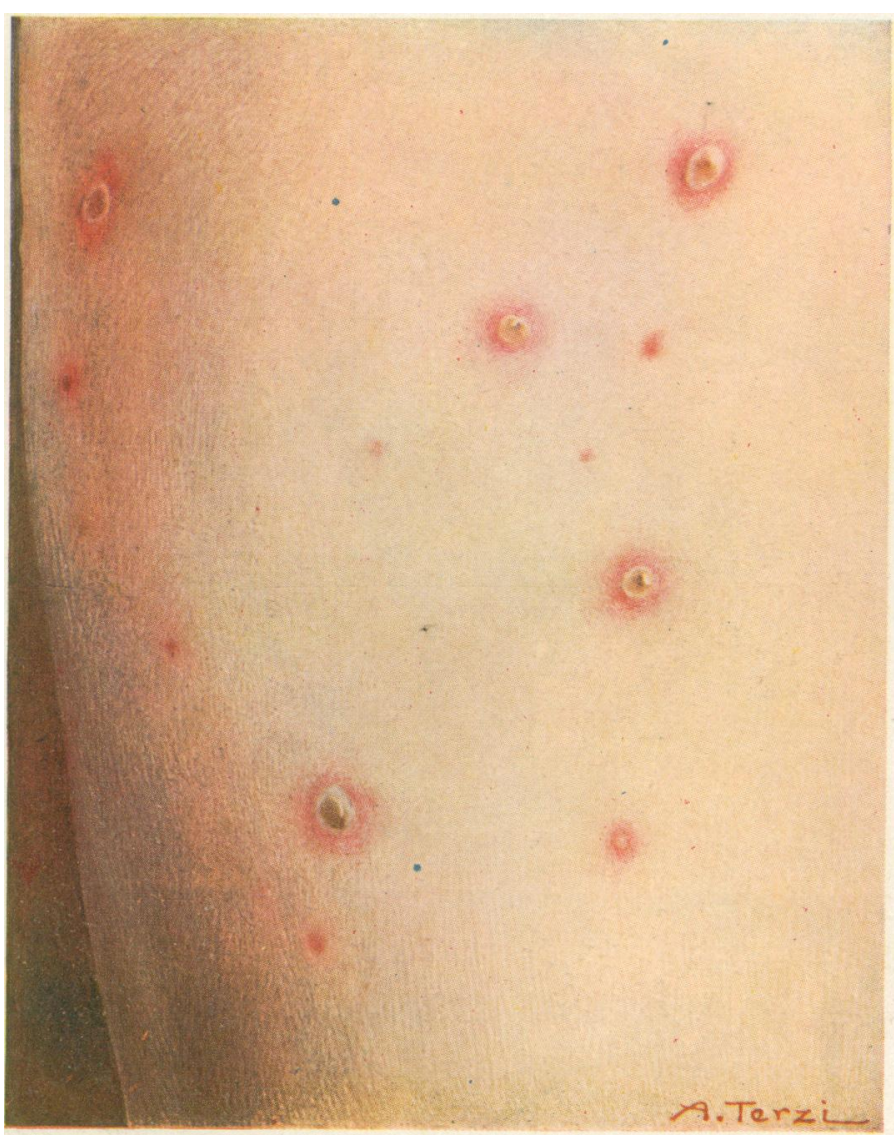

Fig. 3.-CHICKEN-POX. Adult. Second day of eruption.

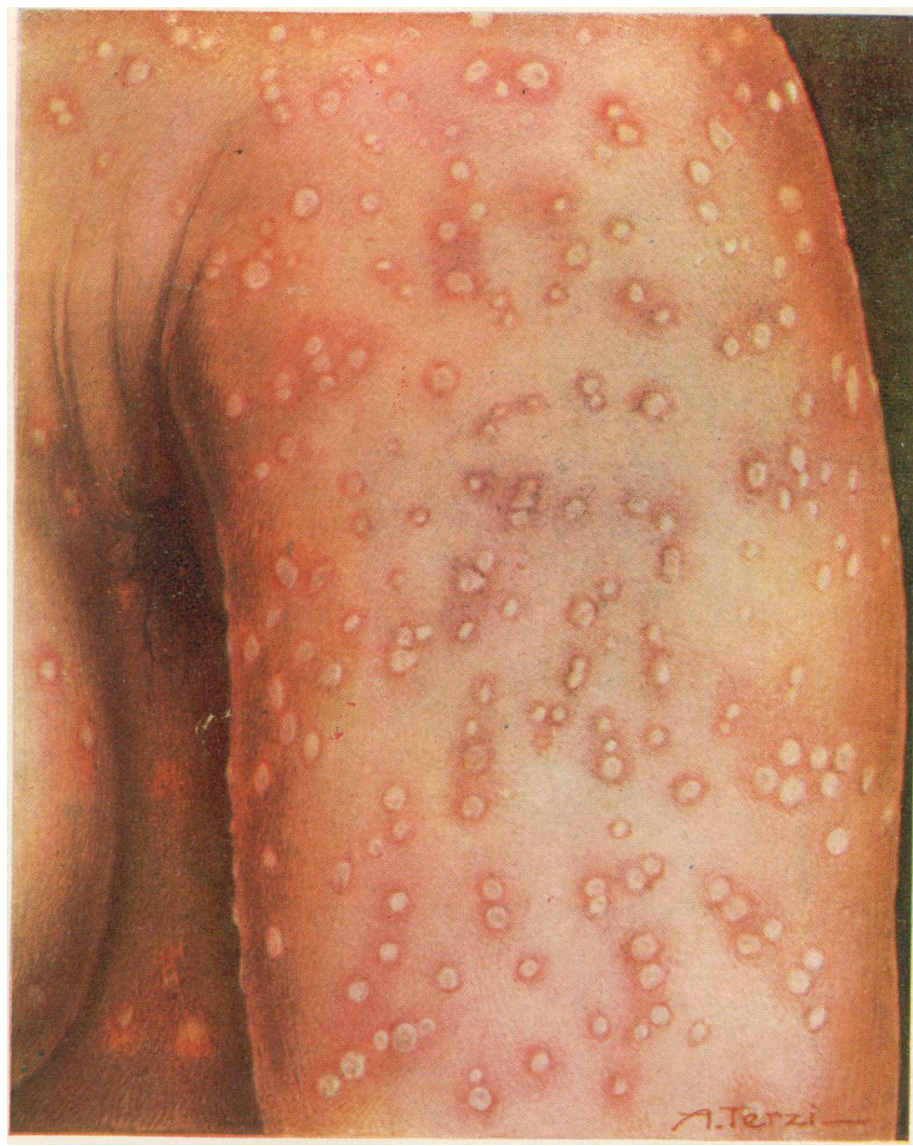

Fig. 2. SMALL-POX - UNMODIFIED. Third to fourth day of eruption.

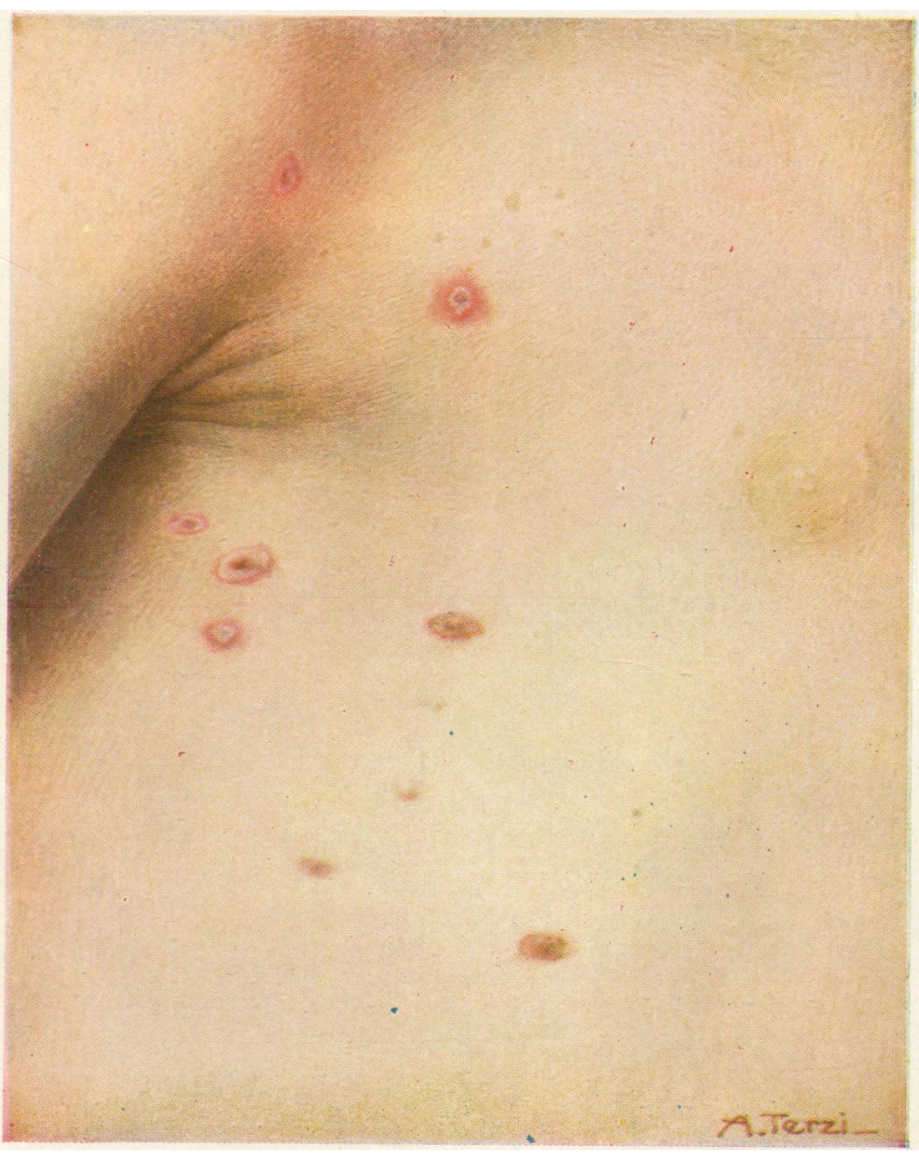

Fig. 4.-CHICKEN-POX. Chlld aged 7. Third day of rash. 


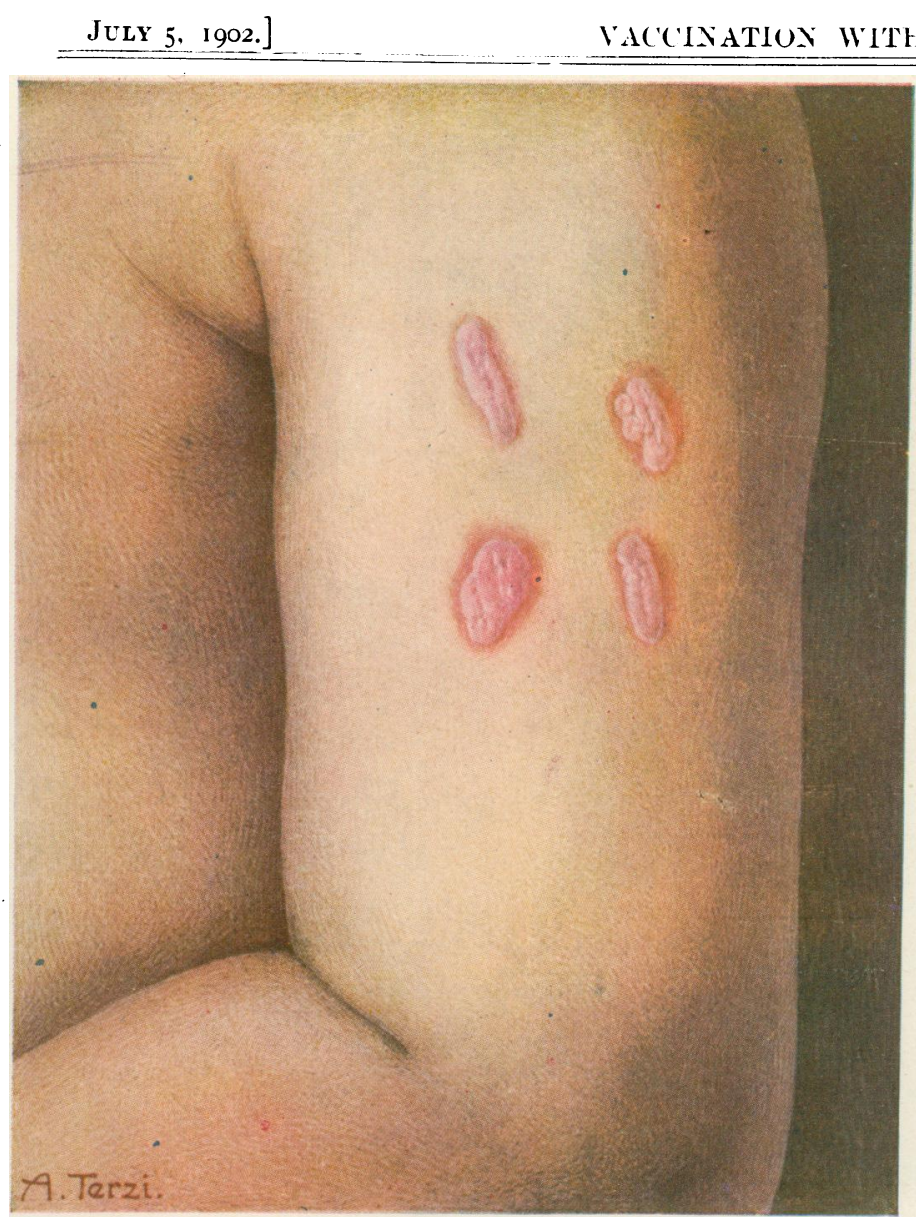

Fig. 5.-VACCINATION. Fifth day.

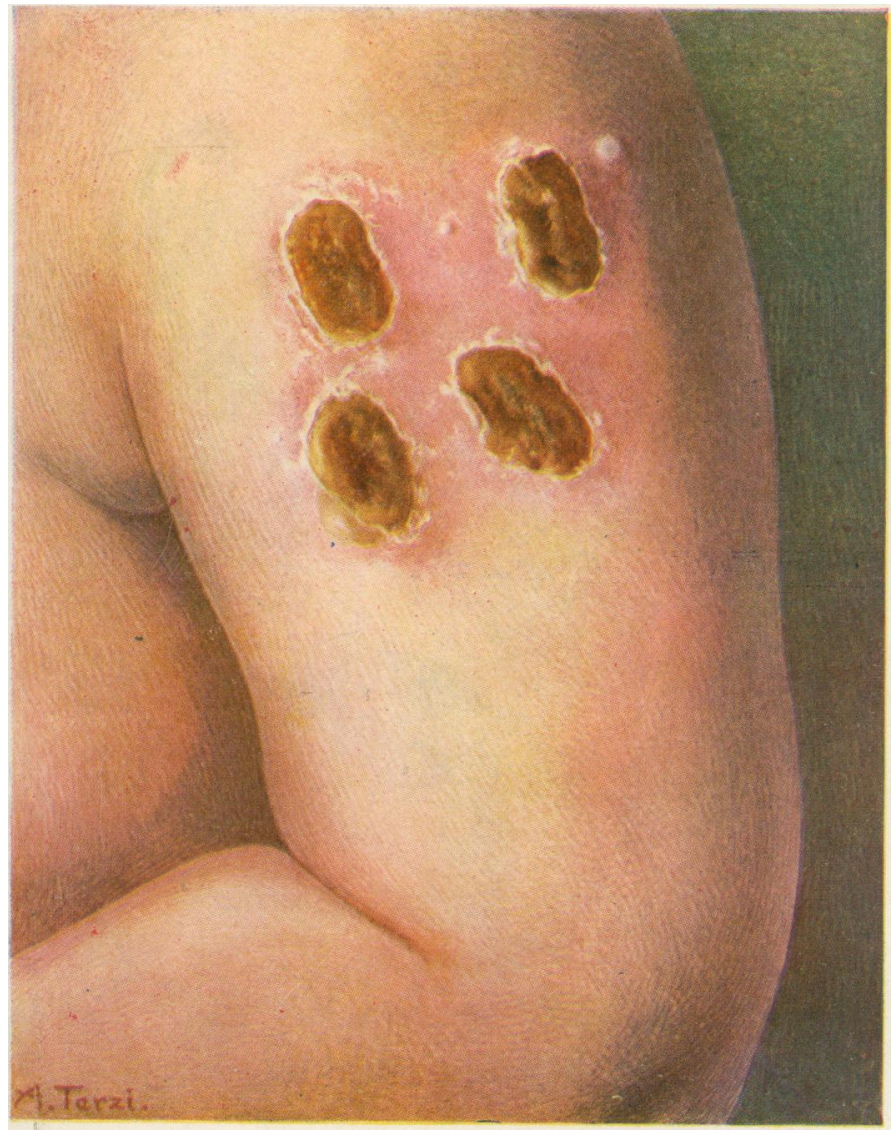

FIg. 7.-VACCINATION. Tweifth day
THE BHITIEH

GLYCERINATED LYMPH

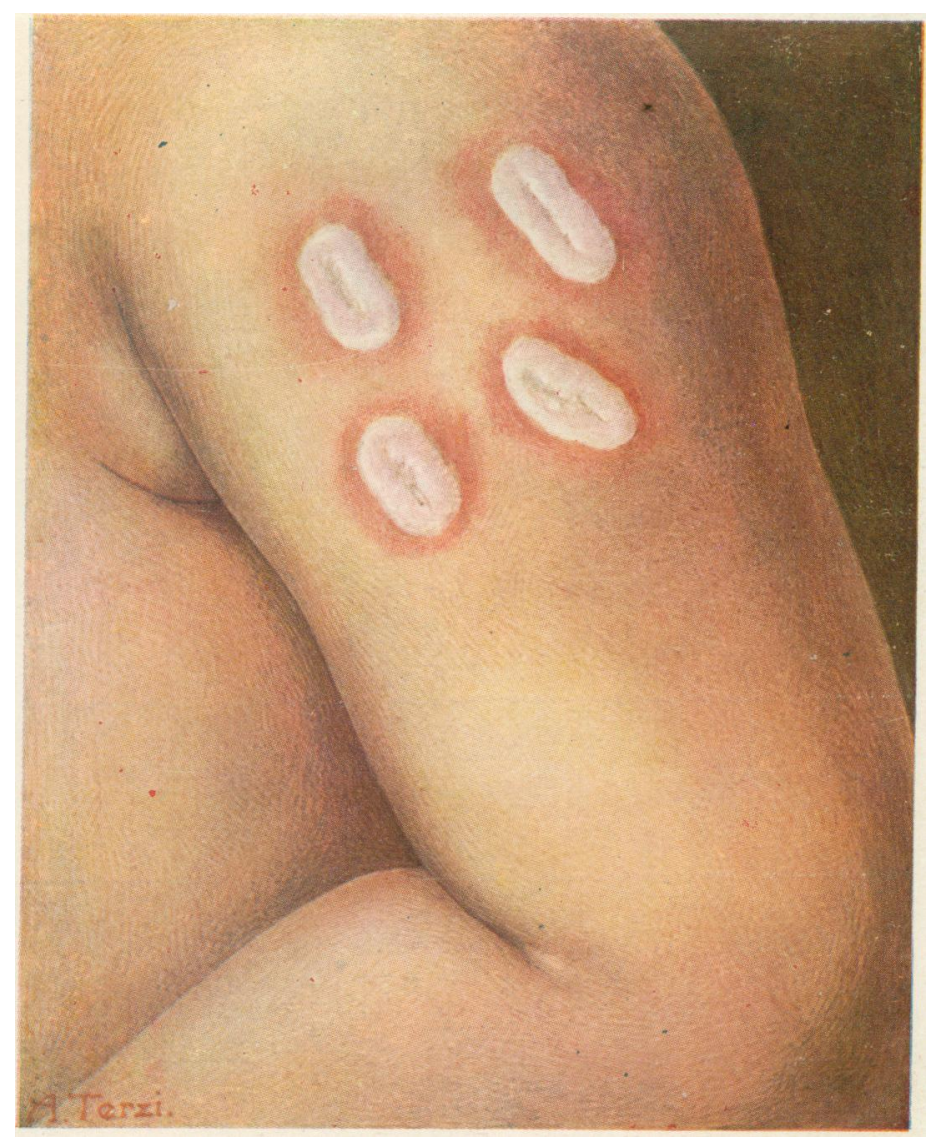

Fig. 6.-VACCINATION. EIghth day.

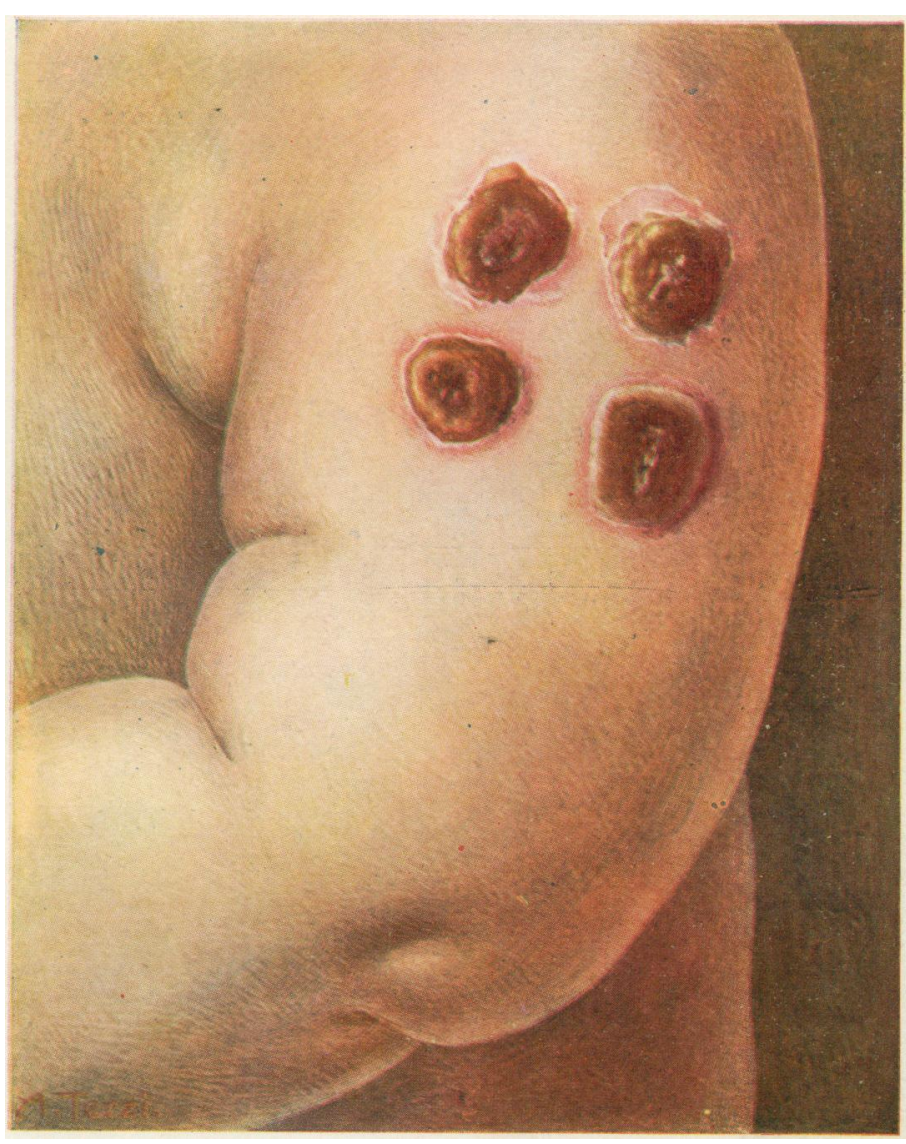

Fig. 8. -VACCINATION. Fifteenth day. 
one tries to get-a tiny point of blood in each scarification. It is also very rare for the scratching to make the child cry. Within two minutes in most cases the edges of the scratches become tumid and on stretching the skin one can distinctly see an urticarial effect. The lancet is wiped with boric wool, sterilized at once, and put back in its case.

Now, what is to be done to protect the places from contamination? The plan which I almost invariably adopt is to take a pad of boric wool 3 in. by 2 in., place it over the vaccinated area with the long axis vertical, and fix it in position by two to to is c.cm. (4 in. to 6 in.) strips of $\frac{1}{3}$ in. rubber adhesive plaster (sold in ten yard spools) making sure that the plaster will be clear of the vesicles, and that the upper strip is fixed solely to the arm, and not to the axillary folds. The boric wool should answer the test of giving a distinct green flame when lighted after soaking with alcohol, but should not be heavily weighted with the antiseptic. This method, however, is not perfect and is simply one among a vast number which have eprung into use within the last few years. We may classify the methods thus-

(a) The antiseptic tissue, film, taffetas, or goldbeaters' skin, one side coated with dextrin or other adhesive material. The skin around the points of vaccination is moistened, and the film pressed over the whole area.

(b) The asepiic pad, contained, with a gauze sponge for washing the arm in a germ-proof bag of gamgee tissue, affixed to the skin by tapes or by an oval of plaster.

(c) The antiseptic pad, which is probably the most popular and generally useful form of dressing.

Hartmann's wood-wool pads have easily led the way in this department, but are being run very closely by other makers. The pad may be impregnated with any ordinary antiseptic, and may be affixed with tapes or plaster. A very useful pad has just been introduced which has a strip of plaster at the top and gauze tapes below, so that the vesicles may be uncovered without removing the pad, and the surface dusted with any soothing powder.

(d) Collodion may be used. M. Chambon, of the Institut de Vaccine Animale, Paris, first removes the excess of glycerinated lymph with weak boric lotion, and then a sterilized brush of cotton wool twisted round a sort of match stalk is dipped into collodion and applied to each insertion.

Tegmin is another preparation recently introduced from Vienna in sterile collapsible tubes, which may be applied directly to the scarifications, and dries rapid]y.

Boric gelatine and similar annlications have their advocates. In all these cases probably an antiseptic pad would in addition be advisable.

So far, then, for our methods of vaccinating. What results may we expect to obtain from the use of glycerinated calf lymph? The results are not, cannot be, uniform, but will depend upon

I. The character and age of the lymph.

2. The idiosyncrasy of the person vaccinated.

3. The state of the person vaccinated in regard to previous vaccination.

It is only possible, therefore, to give general indications as to the course of the development of the vac cination. One may roughly say that the total duration of a normal vaccination from operation to falling of scab is twenty one days; this period being divided into two equal parts corresponding to the evolution and the involution of the resicles. French writers are fond of speaking of a period of incubation followed by the periods of eruption, maturation, desiccation, and cicatrization. The use of the term "incubation" is rather ambiguous, however, as the vaccine has a continuous and progressive effect from the time of its introduction to the Jymph spaces of the skin, and there is no clear demarcation of stages, the word incubation being used simply to express the sum of changes up to the distinctly papular or early vesicular stage. Furthermore, the use of the term incubation in this sense tends to confuse the parallelism which exists between ordinary variola, inoculated variola, and vaccinia, the incubation stage in the two former signifying the period up to the commencement. of constitutional symptoms, a period with a less definite, but none the less real, existence in the case of the last-named.

Thus the incubation stage of small-pox is twelve days, of the inoculated variety eight days, while the general symptoms after vaccination appear usuaily between the sixth and eighth days in the form of restlessness, and slight elevation of temperature, with development of areola around vesicles, and go on in a variable number of cases to the appearance of an exanthem between the ninth and eleventh days. The word incubation therefore, in my opinion, should be reserved for the period up to the commencement of general sy mptoms, and should not be used in the description of the local lesion at all.

There is another word in respect to which ambiguity is apt to arise, that is, the word "day." It should be distinctly understood that when the end of the fifth day is spoken of the end of five complete periods of twenty-four hours is meant. For example, the calf vaccine is collected at the end of five days (120 hours) from the vaccination of the calf.

During the first day or two the results of the mechanical injury are more evident than those of specific infection. The immediate result of the operation is to divide the cuticle, and just scratch the true skin, tearing across small capillarifs and giving rise to tiny haemorrhagic points in the lines of scarification, with vasodilatation and tumefaction which in a minute or two becomes distinctly urticarial in appearance, especially if the skin is put lightly on the stretch. With active lymph the scratch thus made never becomes "dead" like an ordinary scratch, but right from the commencement a little flush is seen, and a little papular elevation is apparent which serve to show that the lymph is taking. In the primary vaccination of children who are old enough to be able to appreciate it a little itching is noticed from the very first day. By the beginning of the third day it is not uncommon to find the scratch in a flat-topped papular condition, like as if a line of lichen ruber planus had been run along the skin. The end of the fourth or beginning of the fifth day will show this panular condition becoming distinctly vesicular with a depressed line in the centre corresponding to the line of scarification. Up to this stage the surface of the vesicle has been a very delicate pale rose pink with a deeper pink skin margin, but at this point the geparation of the epidermis from the vascular part of the skin by the further development of the vesicle gives the surface a bluewhite, opalescent, pearly appearance outside which is the pink ring which increases from the fifth to the tenth day and is called the areola. The breadth of the papulo on the third day is from 1 to $2 \mathrm{~mm}$., and of the vesicle on the fifth day from 3 to $4 \mathrm{~mm}$.

The careful investigation of the histology of the vaccine lesion in the calf as reported by Drs. S. M. Copeman and Gustav Mann in the Supplement to the Twenty-eighth Annual Report of the Local Government Board. has thrown considerable light upon the character of the changes which produre the vesicle which is now formed. Briefly, it may be said that the central depression marks the position of the external and internal clot and local necrosis following the immediate mechanical and chemical injury to the skin tissues by the process of vaccination. The vesiculation is really a distensinn of the intracellular lymph spaces of the Malpighian (prickltcell) layer of the epidermis, groups of epithelial cells being converted into the walls of a sponge-like intercommunicating system of lymph spaces which break into one another, forming bullae filled with vaccine lymph. One would, therefore, no more expect the vesicle to collapee on puncture than one would expect, a sponge soaked in gum to do so. Puncture, nowever, will be followed by the exuration of some of $t ! e$ lymph, which will coagulate on the surface of the vesicle, if left dry, and seal up the wound.

By the seventh day the breadth of the vesicle may be 5 or $6 \mathrm{~mm}$., and we observe that, while the margin still retains its glistening, translucent. pearly character with the thin palered areolar line around it, the rest of the surface presents a more opalescent bluish-white appearance, the base is distinctily indurated as felt between finger and thumb, and the whole vesicle is more prominent, with commencing engorgement of the axillary lymphatic glands and flattening of the axillary hollow. The temperature may be raised a couple of degrees at the end of this day, and this is the point at which it was usual to collect lymph for arm-to-arm vaccination by puncture of the pearly margin of the vesicle. From this point also to the eleventh day the areola develops, first (eigl,th day) as a patchy redness with papular spurs reaching out from the jed margin of the vesicle, like the mountain spurs which buttress a plateau; 
later (tenth day) there may be coalescence of the areolae of the four vesicies, and fusion of the areas of induration which form the bases of the vesicles, so that the separate vesicles cannot be held between finger and thumb, but the whole vaccinated area forms a prominence on the outer side of the arm from which the vesicles stand out.

While the areola is developing important changes are taking place in the vesicle itself. On the ninth day we may notice, in addition to the pearly margin, and the duller bluish-white surface, a dead yellow-white centre, frequently with epidermal débris. This is due to the coagulation and necrosis of the central elements of the vesicle (lymph and cells), and the process thus commenced spreads from centre to circumference, the course of necrosis being marked by deepening coloration from ochrey-yellow, through burnt sienna or burnt umber of deepening shades, with a frequent admixture of purple or violet. brown; to the final black-brown scab, these colour changes being completed usually within the second week (ninth to fifteenth daýs).

The areola attains its maximum on the tenth day, and quickly subsides, leaving a dull purple-red coloration of the skin, with delicate, lace-like desquamation immediately round the vesicles, the induration of the base passing away, also; very rapidly, leaving the skin flat and supple.

The size of the vesicle, on the ninth day, 7 to $8 \mathrm{~mm}$., may reach a maximum of $1 \mathrm{~cm}$. On the tenth or eleventh day, but does not usually advance after that: The pearly edge loses its lustre on the ninth or tenth day, and about this time one frequently observes the desquamation of the superficial layers of ' the vesicular epidermis, which brings the original stratum lucidum to form the surface of the vesicle.

The changes which follow this are not visible, but consist, according to Dr. Cory, of an ingrowth from the stratum lucidum dating from the tenth day, which gradually cuts off the scab at its base. As a matter of fact, were it not for the commencement of immunization, there is no reason why the vesicle should not spread centrifugally until all the skin had been invaded, just as one finds a tendency on the part of all fungoid skin conditions to spread. As soon as immunization commences, the scab begins to be a foreign body, and is removed just in a similar fashion to the mummified umbilical cord when its function has been aceomplished. One occasionally meets with interesting cases in which the immunizing process appears to be delayed, and the vesicle keeps spreading, sometimes with the formation of supplementary vesicles at the peripliery until quite a large area is involved, the appearance being not unlike that figured in some of the plates of inoculated small-pox. Generally in these cases the constitutional symptoms are not more marked than in an ordinary vaccination, and the only danger is that the period of healing is delayed, and thus the opportunities for injury and secondary infection are increased. Dr. Cory found that on the average of a considerable number of cases the scab fell on the twenty-first day, leaving the skin completely healed, and a depressed pink scar, which in the course of a few months became the white vaccination scar with which all are familiar.

It should be added that from the ninth to the twelfth days after vaccination one occasionally sees a rash on the skin, about the nature of which doubt has arisen. While the vaccine roseola may be simply a toxin rash similar to the antitoxic rashes or the rheumatic or digestive erythemata, there is some reason for considering it the true analogue of the secondary rash which appears on the eleventh day after small-pox inoculation.

The course of temperature is well shown in the accompanying chart from a boy of 9 , vaccinated May 16th. 1902, which was kindly obtained for me by the Lady Superintendent of thie Westminster District Nurses.

Such is the course of an ordinary primary vaccination. What difference does the fact of a previous vaccination make?

First, it should be recognized that the immunizing effect of vaccination appears to be much more rapidly destroyed, as would naturally be imagined, during the first fifteen years of life, the period of transition from infancy through childhood to puberty, than in any subsequent period of fifteen years. Thus, while revaccination of a child of io to 15 , successfully vaccinated in infancy, will yield in many cases vesicles of good size, revaccination in an adult, within ten or fifteen years of a successful primary vaccination, will not usually reach more than a papular or very poor vesicular stage. This is important from the point of view of general revaccination, for while a primary vaccination may require repetition within ten or fifteen years, there will not be the same urgency for a second repetition of the operation. Revaccination with the same lymph as produces the effects described above as characteristic of primary vaccination, within five to ten years of previous successful vaccination or small-pox in an adult, gives rise to itching on the first day, the formation of an inflamed irritable elevated scratch on the second and third days with a little axillary tenderness, and by the end of a week all that can be seen is a red line corresponding to the scratch, which may persist for some weeks. Revaccination within ten or iffeen years of a previous successful vaccination gives rise generally in the adult to a distinct papule which may become vesicular, and form a small scab which falls in the second week. If more than fifteen years has elapsed the results were more nearly approximate to a primary vaccination, as the length of interval increases. If however, we are vaccinating for the first time since an infantile vaccination it is possible to get small vesicles at 7 years of age, and large vesicles at 14, especially if the infantile vaccination has been of poor quality. If, however, there are four or five good marks, the vaccination is generally abortive until the twelfth to fourteenth year.

Revaccination presents one or two features of some importance which demand passing notice. First, there is much more tendency to oedema of the distal part of the limb, commencing near the vesicles, and spreading with definite ridge-like margin to just above the elbow, then just over the origin of the supinators, then the mid-forearm, and, lastly wrist and even hand. This oedema subsides, in some instances very suddenly, especially if one orders the hand to be supported by a sling, and foments the axilla with hot water, for the axillary glands appear to be the key of the situation. It is only important because it is sometimes mistaken for cellulitis. It is not uncommon to find the general symptoms taking on an influenzal or rheumatic character, and in one or two cases temporary cardiac souffes have been audible, the whole passing off with simple antifebrifuge treatment. It is important to recognize that revaccination after twenty years is generally accompanied with constitutional symptoms, which demand the same kind of care as if one had a bad cold. It is one of the strongest arguments for compnlsory revaccination at the close of school life that, if left till adult life is reached, revaccination is a distinctly more troublesome business, and it is frequently impossible to take the precautions which prudence would suggest.

The same lymph, then, will produce very different results according to the state of the patient in respect of previous vaccination or small-pox, and it is very useful to check the results of revaccination by a parallel series of primary vaccinations, so that we may have assurance as to the activity of the lymph used. For it is equally true that lymph. may be variable, and variable results may own this as their cause, a veritable fons et origo mali. Inert lymph-how much it has had to answer for. The certificates of insusceptibility, which in the last annual return numbered over 3,000 , are almost all to be put down to its credit. It is not yet sufficiently widely known that if one vaccinates calf or child with inert glycerinated calf lymph, lymph which has even been sterilized by heat, there is apparently an absorption of toxin sufficient to temporarily inhibit the success of a further vaccination even with active lymph. The practice, therefore, of vaccinating immediately upon evidence of failure of a first vaccination is almost certain to lead to failure a second time. It is necessary to wait at least one month, and preferably two months before making a second attempt. It is questionable whether the temporary immunity extends to protection against smallpox.

Lymph of feeble activity is also responsible for the group of cases known by French authors as "vaccine rouge," in which, in place of the normal vesicle, a fleshy, red, naevoid tubercle appears on the site of vaccination, which passes off in the course of a week or month, but which appears to have no protective influence. 
I have not made any reference to the complications which may mark the course of a vaccination and their treatment. The various methods of protecting the vaccine vesicle have very largely diminished the risks of the operation, a result which was anticipated in the report of the Royal Commission. If only the vesicle can be kept free from mechanical injury, or, when injured, if exudation can be checked and the ingress of organisms prevented until the scab, kept perfectly dry, has fallen, leaving the typical scar, we shall have little fear of any complications.

One method which I have found of exceptional value, and which I believe is worthy of wide adoption, is the use of a saturated aqueous solution of picric acid to paint over the vesicles and surrounding skin, wherever there has been any such injury to the vesicles. I was led to try this from an observance of the value of the same application in the somewhat similar condition after burns and scalds. The application is painless, and I have simply covered it with antiseptic wool, leaving it untouched for several days.

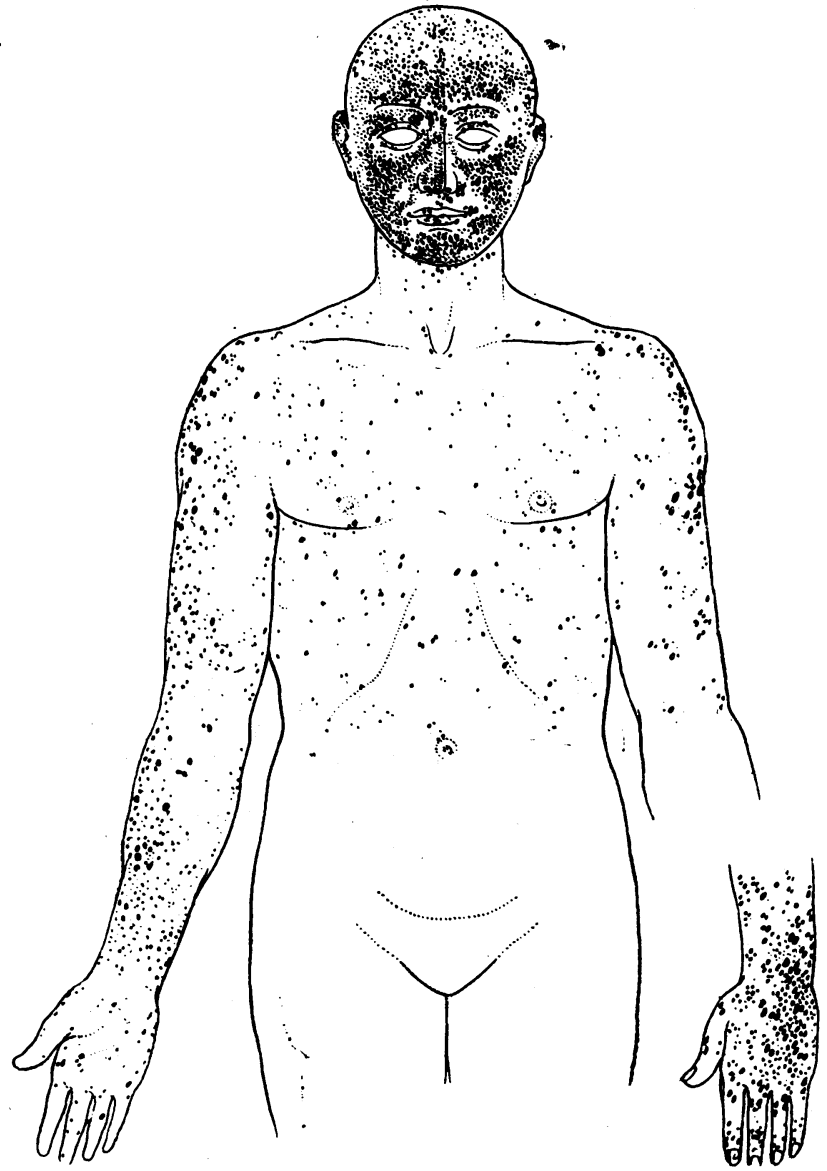

Fig. 1.-Diagram showing distribution of eruption in small-pox. (To illustrate Dr. Wanklyn's paper.)

In conclusion, it is necessary to add that the description of the course of the vaccination is an attempt to give a composite picture of the course of a process which is distinctly variable, both by acceleration and retardation and in intensity, and that it is gleaned from the use of the lymph issued by the National Vaccine Establishment, which has been remarkably uniform in its results for some time.

While the writer is probably as sensible as any one of the lacunae which remain to be filled up, his main object has been to open up two lines of investigation to which every one who vaccinates may contribute some facts from his own ex. perience, by the pursuit of which the risks of the operation may be reduced to a minimum, and yet which are but scantily represented in easily accessible medical literature.
Description of Coloured Plates Illustrating Dr. Cope's Paper. (Between pages 44 "and 45. .)

Fig. 5-Child, W. I male, aged 6 months. Appearance at beginning of fifth day. Situation of individual scratches indicated by depressions in the developing vesicles. Faint flush round each vesicle.

Fig. 6.-Child, D. C., female, aged ro months. Appearance at beginning of ninth day, but somewhat delayed, and would pass quite well for eighth day, except that surface of vesicles is dull.

Fig. 7.-Same child, D.CC. Appearance at end of twelve days. "Two supplementary vesicles on arm. Course of development unusually rapid between ninth and twelfth days in this case, desquamation being active around vesicles, the surfaces of which have darkened right to the edge, though there are patches where the vesicular edge is still visible.

Fig. 8.-Child, J. S., male, aged 4 months. Appearance on fifteenth day. The lower right-hand vesicle has not advanced so rapidly as the rest, and shows more typically than Fig. 3 the nore usual method of desiccation of the vesicle.

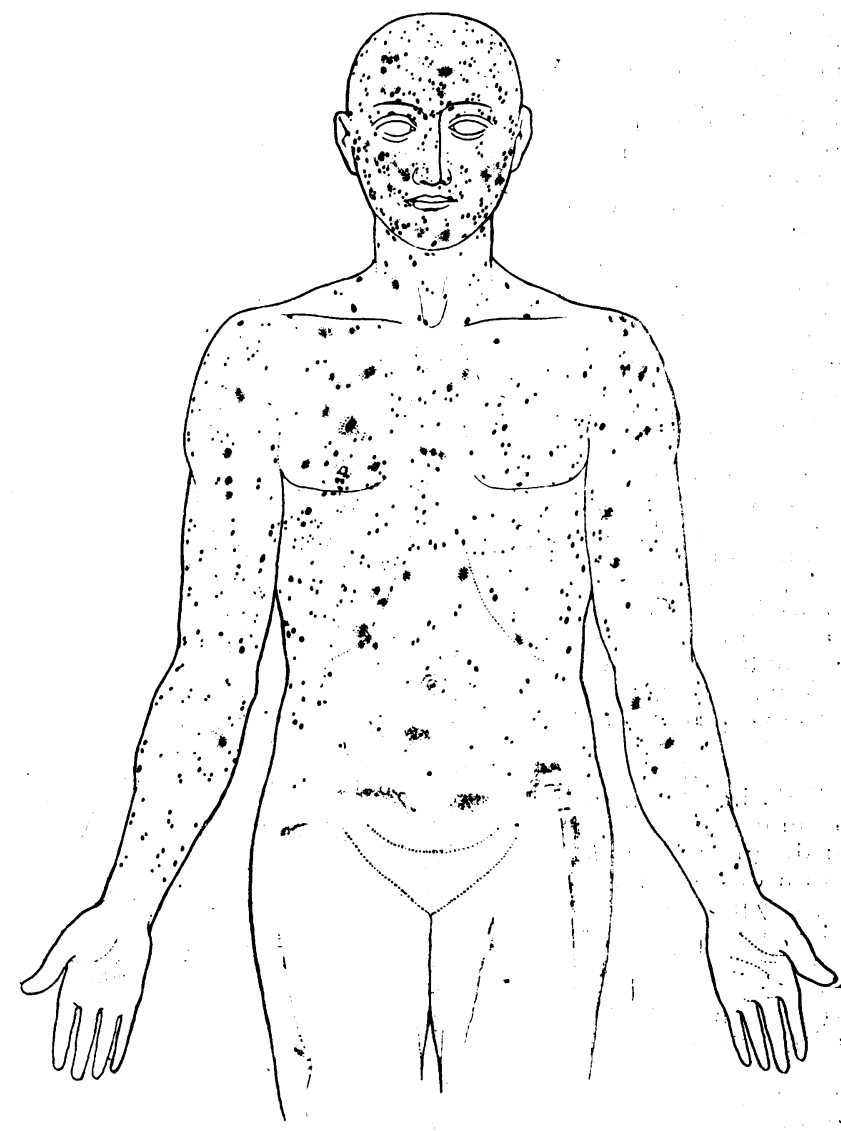

Fig. 2.-Diagram showing distribution of eruption in chicken-pox (To illustrate Dr. Wanklyn's paper.)

\section{DIFFERENTIAL DIAGNOSIS BETWEEN VARIOLA} AND VARICELLA.

By W. McCONNEL WANKIYYN, B.A.CANTAB., M.R.C.S., L.R.C.P.,

Small-pox;Referee and Medical Superintendent of the River A bulance Service of the Metropolitan Asylums Board.

Few problems in diagnosis have heen of $r$ ore practical importance recently than the differentiation between variola and varicella. The following remarks are based upon the observation of 200 cases of varicella sent to the diagnosing station of the Asylums Board during the recent outbreak of small-pox. Since these cases were sufficiently puzzling to be taken for small-pox, it. will be of interest to touch upon the distinctive features of the two diseases, and indicate those which led to a correct diagnosis being made. 